\title{
Experimental investigation of the saturated regime of short pulse amplification in counter-pumped Raman amplifiers
}

\author{
Guillaume Vanderhaegen ${ }^{1}$, Pascal Szriftgiser ${ }^{1}$, Matteo Conforti ${ }^{1}$, Alexandre Kudlinski ${ }^{1}$, Arnaud Mussot ${ }^{1}$ \\ 1. University of Lille, CNRS, UMR 8523 - PhLAM - Physique des Lasers Atomes et Molécules, F-59000 Lille, France
}

With the development of high-speed optical telecommunications, also appeared optical amplifiers such as Raman amplifiers [1,2]. Allowing a distributed amplification and offering a wider gain bandwidth than other amplification techniques, there are preferably used in their counter-pumped regime (relative intensity noise transfer reduced). In the case of a pulsed signal to amplify, it is important to understand the transient effects of such an amplifier [3]. In this paper, the influence of short pulses width on transient and saturation effects of a counter-pumped Raman amplifier is thus experimentally reported.

The experimental setup is displayed in Fig. 1. (a). A $1550 \mathrm{~nm}$ laser is first shaped into a train of $T_{S}$ width pulses with a $4.9 \mathrm{kHz}$ repetition rate (the pump is then undepleted at each new pulse generation). They are then amplified with an erbium doped fiber amplifier and filtered (AO modulator) before being injected into a $20.3 \mathrm{~km}$ long NZDSF. A counter-propagating Raman pump wave located 13.2 THz away to benefit from the maximum Raman gain, amplifies these pulses within the fiber. The amplified pulses are then captured by a photodiode at the fiber output. The ON/OFF gain is measured for different signal pulse widths ranging from $T_{S}=10 n s$ to $T_{S}=4 \mu s$ to highlight the influence of the pulse duration on the amplifier saturation. The Raman pump power is set to $P_{R}=898 \mathrm{~mW}$ and the signal peak power to $P_{S}=25 \mathrm{~mW}$. The results are displayed on Fig. 1. (b)-(d). In the
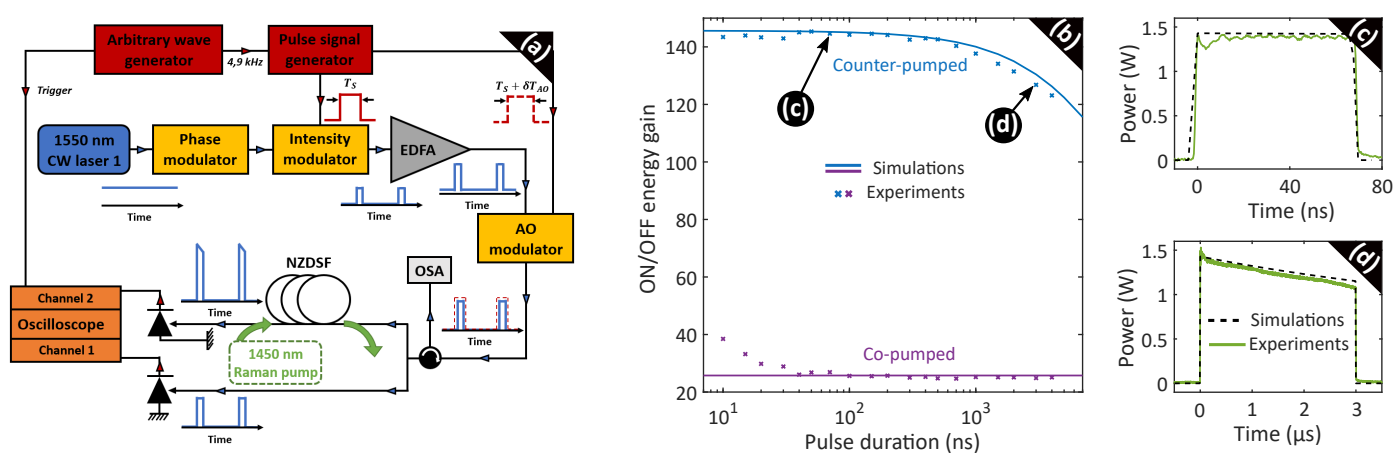

Figure 1: (a) Experimental setup. (b) Energy gain as function of the signal pulse width for an initial Raman pump power $P_{R}=898 \mathrm{~mW}$ for counter (blue) and co-pumped (purple) Raman amplifiers. (c)-(d) Amplified pulses time profiles for $T_{S}=70 \mathrm{~ns}$ and $T_{S}=3 \mu \mathrm{s}$ for the co-pumping amplifier.

counter-pumping configuration, for short pulse widths $(<100 \mathrm{~ns})$, the gain is equal to the unsaturated gain of the amplifier in the CW approximation [1]. The output peak power of these signals is almost twice the Raman pump power. This surprising feature is due to the facts that (i) the pump and signal waves counter-propagate and (ii) the signal is pulsed. Thus, photons transferred from the pump to the signal belong to different pump time slots. This number remains negligible compared to the total number located in the pump, leading to a constant Raman gain all along the fiber length (regardless the losses). The amplifier then never saturates, the pulses are thus linearly amplified without distortion (Fig. 1. (c), solid line) even above the Raman pump power. By increasing the pulse duration, the photon number becomes more significant, leading the amplifier to saturate. Therefore, the gain drops for these longer pulse durations and pulses are distorted ((Fig. 1. (d), solid line). For comparison, the gain curve recorded from a co-propagating configuration is shown in violet line in Fig. 1. (b). Here, as the pump and signal waves almost propagate at the same speed, pump photons originate from the same time slot leading the amplifier to saturate for any pulse duration and the gain to remain times weaker than in the counter-propagating configuration. Numerical simulations shown in solid lines for gain curves (Fig. 1. (b)) and black dashed lines for pulses time profiles (Fig. 1. (c)-(d)) are in excellent agreement with experiments.

To conclude, we reported the first experimental demonstration of the influence of the signal pulse duration on the saturation process in counter-pumped Raman amplifiers. For short pulses signals, we revealed that the output peak signal power can be larger than the Raman pump power.

\section{References}

[1] C. Headley and G. Agrawal, Raman Amplification in Fiber Optical Communication Systems (Academic Press, 2005).

[2] 1. R. G. Smith, "Optical Power Handling Capacity of Low Loss Optical Fibers as Determined by Stimulated Raman and Brillouin Scattering," Appl. Opt. 11, 2489 (1972).

[3] V. V. Kozlov, S. V. Manakov, and S. Wabnitz, ”Exact solution for the gigantic amplification of ultrashort pulses in counterpumped Raman amplifiers," Opt. Lett. 36, 1632 (2011). 